\title{
Urge urinary incontinence was associated with increased risk of falls and non-spinal, non-traumatic fractures in older women
}

Brown JS, Vittinghoff E, Wyman JF, et al for the Study of Osteoporotic Fractures Research Group. Urinary incontinence: does it increase risk for falls and fractures? J Am Geriatr Soc 2000 Jul;48:721-5.

QUESTION: In community dwelling older white women, does weekly or more frequent urge and stress urinary incontinence increase risk of falls and non-spinal, non-traumatic fractures?

Design

Cohort study with mean follow up of 3 years (Study of Osteoporotic Fractures [SOF]).

Setting

4 clinical care centres in Maryland, Minnesota, Oregon, and Pennsylvania, USA.

\section{Participants}

6049 community dwelling, ambulatory, white women who were $\geqslant 65$ years of age (mean age $79 \mathrm{y}$ ), attended 5 SOF clinic or home visits, completed a physical examination and self administered questionnaire, provided data on urinary incontinence, and returned $\geqslant 1$ postcard reporting falls after visit 5 . Institutionalised women were excluded.

\section{Assessment of risk factors}

Live births; hysterectomy status; smoking status; alcohol use; walking; total weekly excursions outside of the home; medical history including hip or knee replacement, stroke, chronic obstructive pulmonary disease, diabetes, Parkinson's or Alzheimer's disease, or arthritis; self reported joint pain; falls within the past year; functional status; medication use; and cognitive function. Frequency and type (urge, stress, or mixed) of urinary incontinence were assessed at the fifth clinic visit.

\section{Main outcome measures}

Reported falls and non-spinal, non-traumatic fractures (fractures were confirmed by radiographs).

\section{Main results}

$25 \%$ of women reported $\geqslant 1$ weekly episode of urge incontinence and $19 \%$ reported $\geqslant 1$ weekly episode of stress incontinence; $12 \%$ reported both types of incontinence. Women with no incontinence or less than weekly incontinence were the control group. $55 \%$ of women reported $\geqslant 1$ fall during the mean 3 years of follow up and $8.5 \%$ reported $\geqslant 1$ fracture. After adjusting for age and other potential confounding factors, urge incontinence was associated with falls $(\mathrm{p}<0.001)$ and fractures

Source of funding:

National Institute on

Aging.

For correspondence:

Dr J $S$ Brown,

Department of Obstetrics,

Gynecology, and

Reproductive Sciences,

University of California,

San Francisco, 2330 Post

Street, Suite 220, San

Francisco, CA 94115 ,

USA. Fax +1415353

9509 .

A modified version of this abstract appears in ACP Journal Club. $(p=0.02)$, whereas stress incontinence was not $(p \geqslant 0.3)$

Risk of falls and non-spinal, non-traumatic fractures in women with $\geqslant$ weekly urinary incontinence at a mean follow up of 3 years

\begin{tabular}{lll} 
Outcomes & $\begin{array}{l}\text { Type of } \\
\text { incontinence }\end{array}$ & Odds ratio $(95 \% \mathrm{Cl})$ \\
\hline Falls & Urge & $1.26(1.14$ to 1.40$)$ \\
\hline & Stress & $1.06(0.95 \text { to } 1.19)^{*}$ \\
& & Relative hazard $(\mathrm{Cl})$ \\
\hline Fractures & Urge & $1.34(1.06$ to 1.69$)$ \\
\hline & Stress & $0.98(0.75 \text { to } 1.28)^{*}$ \\
\hline${ }^{*}$ Not significant. & &
\end{tabular}

(table). A dose response relation existed for urge incontinence: a 35\% increase in the risk of falling existed with daily urge incontinence compared with a $21 \%$ increase in risk with weekly urge incontinence.

\section{Conclusion}

In community dwelling older white women, weekly or more frequent urge but not stress urinary incontinence increased the risk of falls and non-spinal, non-traumatic fractures.

\section{COMMENTARY}

The study by Brown et al helps to untangle the controversy about urinary incontinence as a risk factor for falls. They found that weekly or more frequent urge incontinence independently increased the risk of falls by $26 \%$ and the risk of fractures by $34 \%$. In an accompanying editorial, Wolf $e t$ al elaborate a potential causal hypothesis explaining the results. ${ }^{1}$ Older adults have a reduced capacity to divide their attention. Thus, an elderly person who is focused on the urgent need to use the toilet may become inattentive to environmental hazards or postural control, increasing their risk of falling.

Consistent with previous research, the authors included only those fractures that occurred as a result of minimum trauma. The authors argue convincingly that there was probably no differential bias in reporting of falls or fractures in the 2 comparison groups. However, the authors do not distinguish between those falls that resulted in a fracture and spontaneous fractures which, in turn, contributed to a fall. This distinction is especially important in studies of osteoporotic women. The use of a severity scale to assess other injuries resulting from falls would be a useful adjunct. Future research should examine more fully the relation between the concurrent use of psychotropic drugs and diuretics, the presence of environmental hazards, and the timing and location of fall events. The inclusion of non-osteoporotic women and men in future research should be considered.

Important clinical implications arise from this study. Findings support (1) the inclusion of questions on fall risk assessment protocols to differentiate between stress and urge urinary incontinence; (2) among women with urge incontinence, a periodic review of benzodiazepine use, and working with clients and physicians to identify nonpharmacological alternatives for insomnia; and (3) the use of environmental hazard checklists during clinic or home visits to identify hazards that are related to toileting practices (eg, lack of toilet grab bars in the bathroom or obstructed and unlit routes to the toilet).

Nancy Edwards, RN, PhD Associate Professor, School of Nursing University of Ottawa

Ottawa, Ontario, Canada

1 Wolf SL, Riolo L, Ouslander JG. Urge incontinence and the risk of falling in older women.J Am Geriatr Soc 2000;48:847-8. 